\title{
General Topos Semantics for Higher-Order Modal Logic
}

\author{
Steve Awodey ${ }^{1}$, Kohei Kishida ${ }^{2}$, and Hans-Christoph Kotzsch ${ }^{3 *}$ \\ ${ }^{1}$ Carnegie Mellon, IAS \\ awodey@cmu.edu \\ 2 ILLC, University of Amsterdam \\ kishidakohei@gmail.com \\ 3 Ludwig-Maximilians-Universität München \\ kotzsch@lrz.uni-muenchen.de
}

Topos-theoretic semantics for modal logic usually considers structures induced by a surjective geometric morphism $f: \mathcal{F} \rightarrow \mathcal{E}$. $f$ restricts to an injective (complete) distributive lattice homomorphism

$$
\Delta_{A}: \operatorname{Sub}_{\mathcal{E}}(A) \longrightarrow \operatorname{Sub}_{\mathcal{F}}\left(f^{*} A\right),
$$

for each $A$ in $\mathcal{E}$ and natural in $A$ w.r.t. to pullback. Each $\Delta_{A}$ has a right adjoint $\Gamma_{A}$ that composes with $\Delta_{A}$ to an endofunctor $\Delta_{A} \Gamma_{A}$ on the Heyting algebra $\operatorname{Sub}_{\mathcal{F}}\left(f^{*} A\right)$ that satisfies the axioms for an $\mathrm{S} 4$ modality on $\operatorname{Sub}_{\mathcal{F}}\left(f^{*} A\right)$. [3, 8, 9, 11]

Equivalently, regarding $f_{*} \Omega_{\mathcal{F}}$ as an internal frame in $\mathcal{E}$, one has internal adjoints $i \dashv \tau$,

$$
\tau: f_{*} \Omega_{\mathcal{F}} \leftrightarrows \Omega_{\mathcal{E}}: i
$$

where $i$ is the unique frame map from the initial frame $\Omega_{\mathcal{E}}$, and $\tau$ is the classifying map of $f_{*}(T)$, with $1: T \rightarrow \Omega_{\mathcal{F}}$ the subobject classifier in $\mathcal{F}$. [7, 11]

The typical example is a geometric morphism of the form

$$
\text { Sets }^{|\mathrm{K}|} \longrightarrow \text { Sets }^{\mathrm{K}}
$$

for a preorder $\mathbf{K}$, induced by the inclusion $|\mathbf{K}| \rightarrow \mathbf{K}$ of the underlying set into $\mathbf{K}$. This yields "Kripke sheaf" semantics for modal logic. Here $\mathbf{K}$ is a preordered set of worlds, and each functor $P: \mathbf{K} \rightarrow$ Sets gives a domain $P(k)$, for each $k \in \mathbf{K}$, with suitable comparison maps $P(k) \rightarrow P(l)$, whenever $k \leq l$ in $\mathbf{K}$. In this way each functor $P$ determines a "Kripke sheaf", a first-order Kripke frame with varying domains of individuals. 2, 4, 12, Further examples include sheaf structures from the geometric morphism Sets $/ X \rightarrow \operatorname{Sh}(X)$, for a topological space $X$. [1, 5, 6]

In this talk, we will provide a slightly more general algebraic framework of modal structures in an arbitrary topos $\mathcal{E}$ with respect to which it is possible to build models of a certain system of (intuitionistic) higher-oder modal logic. The "geometric" models from before arise as a special case of the latter.

The data for such structures consist first of all of a complete internal Heyting algebra $H$ in $\mathcal{E}$. Since $\Omega_{\mathcal{E}}$ is the initial frame in $\mathcal{E}$, there exists a unique frame homomorphism $i: \Omega_{\mathcal{E}} \rightarrow H$ that has a right adjoint $\tau$, namely the classifying map of the top element $T: 1 \rightarrow H$ of $H$ (cf. [11, 10. In what follows we will consider only those Heyting algebras $H$ in $\mathcal{E}$ such that $i$ is monic.

$H$ interprets the logical operations via its Heyting structure, just as in standard algebraic semantics. Moreover, universal and existential quantification is modelled by indexed meets and

* Research supported by the Alexander-Humboldt Foundation 
joins in $H$, respectively, since $H$ is assumed to be complete. For any object $I$ in $\mathcal{E}, I$-indexed meets are defined to be given by a right adjoint

$$
\forall I: H^{I} \rightarrow H
$$

to the canonical map $\Delta_{I}: H \rightarrow H^{I}$ that arises in turn from the unique map $I \rightarrow 1$ under the functor $H^{(-)}$. $I$-indexed joins are defined as a left adjoint to $\Delta_{I}$.

A model in $H$ is then given by objects $M$ for each basic type in the language, while complex types are interpreted as usual using the cartesian closed structure in $\mathcal{E}$. The propositional type Prop is interpreted by $H$. The interpretation of non-modal formulas is the standard one except for the treatment of equality which is new. Each formula $\varphi\left(x_{1}, \ldots, x_{n}\right)$ in the free variables $x_{1}, \ldots, x_{n}$ is recursively assigned a map $M_{1} \times \cdots \times M_{n} \stackrel{\llbracket \varphi \rrbracket}{\longrightarrow} H$, where $M_{i}$ interprets the type of $x_{i}$. For instance, $\forall y \varphi(x, y)$ is the map $X \stackrel{\lambda_{Y} \llbracket \varphi \rrbracket}{\longrightarrow} H^{Y} \stackrel{\forall_{Y}}{\longrightarrow} H$, where $\lambda_{Y} \llbracket \varphi \rrbracket$ is the exponential transpose of $\llbracket \varphi \rrbracket$, and $X, Y$ interpret the respective types. The map $i \circ \tau: H \rightarrow H$ interprets the modal operator which satisfies the S4 laws by virtue of properties of the adjunction.

More importantly, for each type in the language, equality on the corresponding object $M$ is given by the map

$$
M \times M \stackrel{\delta_{M}}{\longrightarrow} \Omega_{\mathcal{E}} \stackrel{i}{\rightarrow} H,
$$

where $i$ is the unique frame map from before, and $\delta_{M}$ is the classifying map of the diagonal $\left\langle 1_{M}, 1_{M}\right\rangle$.

It can be shown that when $H=f_{*} \Omega_{\mathcal{F}}$, for a geometric morphism $f$, then this definition of model coincides with the standard one associated with $f$. In particular, for equality we have

Lemma. For any geometric morphism $f: \mathcal{F} \rightarrow \mathcal{E}$, and any object $M$ in $\mathcal{E}$,

$$
i \circ \delta_{M}=\overline{\delta_{f^{*} M}},
$$

where $\overline{\delta_{f^{*} M}}$ is the transpose (along $f^{*} \dashv f_{*}$ ) of the classifying map of the diagonal on $f^{*} M$.

This yields the following result, based on an already known fact for the geometric case [13]:

Proposition. The extensionality principles

$$
\begin{gathered}
\forall_{x}(f(x)=g(x)) \vdash f=g, \\
(p \Leftrightarrow q) \vdash p=q .
\end{gathered}
$$

fail to be valid in the algebraic semantics described above.

The reason is precisely the interpretation of equality. However, we have

Proposition. The following statements are valid in the algebraic topos semantics:

$$
\begin{gathered}
\square \forall_{x}(f(x)=g(x)) \vdash f=g, \\
\square(p \Leftrightarrow q) \vdash p=q .
\end{gathered}
$$

As a result, the higher-order $S_{4}$ system that results from replacing propositional and functional extensionality by their modalized versions, respectively, is sound w.r.t. algebraic models in $\mathcal{E}$. 
We will outline in detail why the non-modal versions fail and why the boxed versions are valid in the semantics. It is precisely the map $\tau: H \rightarrow \Omega$ from before which does the relevant work to repair the failure of the standard extensionality principles. In a way, thus, the modal operator as treated here turns out to be precisely what needs to be added to soundly interpret intuitionistic higher-order logic in a complete Heyting algebra in $\mathcal{E}$.

We conjecture, moreover, that this yields a complete semantics w.r.t. toposes for the outlined system of higher order S4 modal logic with the standard extensionality principles replaced by the modalized ones displayed above.

We conclude the talk by observing that every algebraic model in $\mathcal{E}$ arises from a geometric morphism from a suitable topos $\mathcal{F}$, i.e. $H=f_{*} \Omega_{\mathcal{F}}$, for $\mathcal{F}$ the topos of internal sheaves on $H$ and $f$ the canonical geometric morphism into $\mathcal{E}$.

\section{References}

[1] Steve Awodey and Kohei Kishida. Topology and Modality. The Topological Interpretation of First-Order Modal Logic. Review of Symbolic Logic, 1(2):146-166, 2008.

[2] Silvio Ghilardi. Presheaf Semantics and Independence Results for Some Non-Classical First-Order Logics. Archive for Mathematical Logic, 29:125-136, 1989.

[3] Silvio Ghilardi and Torben Braüner. First-Order Modal Logic. In Johan van Benthem and Patrick Blackburn, editors, Handbook of Modal Logic. Elsevier, 2006.

[4] Silvio Ghilardi and G Meloni. Modal and Tense Predicate Logic. Models on Presheaves and Categorical Conceptualization. In Categorical Algebra and its Applications. Proceedings of a Conference, held in Louvain-La-Neuve, Belgium, July 26-August 1, 1987, Lecture Notes in Mathematics, vol. 1348, pages 130-142. Berlin: Springer, 1988.

[5] Kohei Kishida. Neighborhood-Sheaf Semantics for First-Order Modal Logic. Electronic Notes in Theoretical Computer Science, 278:129-143, 2011.

[6] Koheii Kishida. Topological Completeness of First-Order Modal Logic. In Thomas Bolander, Torben Braüner, and Silvio Ghilardi, editors, Advances in Modal Logic, volume 9, pages 1-17, 2012.

[7] R Lavendhomme, Th Lucas, and Gonzalo Reyes. Formal Systems for Topos-Theoretic Modalities. Bulletin de la Societé Mathematique Belgique, 41 (Ser A):333-372, 1989.

[8] Michael Makkai and Gonzalo Reyes. Completeness Results for Intuitionistic and Modal Logic in a Categorical Setting. Annals of Pure and Applied Logic, 72:25-101, 1995.

[9] Gonzalo Reyes. A Topos-Theoretic Approach to Modalities. Note Dame Journal of Formal Logic, 32:359-391, 1991.

[10] Gonzalo Reyes and Marek Zawadowski. Formal Systems for Modal Operators on Locales. Studia Logica, 52:595-613, 1993.

[11] Gonzalo Reyes and Houman Zolfaghari. Topos-Theoretic Approaches to Modalities. In Category Theory. Proceedings of the International Conference held in Como, Italy, July 22-28, 1990, Lecture Notes in Mathematics, vol. 1488, pages 359-378. Berlin: Springer, 1991.

[12] Shehtman Valentin and Dimitrij Skortsov. Semantics of Non-Classical First-Order Predicate Logic. In P Petkov, editor, Mathematical Logic, pages 105-116. New York, 1990.

[13] Hans-Jörg Winkler. Higher-Order Modal Logic in a Categorical Setting. Diploma Thesis. EberhardKarls-Universität Tübingen, 2012. 